\title{
Community of general scientists
}

\section{Juno spacecraft}

\section{Vladis Jefferson}

Published on: Jun 11, 2021

License: Creative Commons Public Domain Dedication (CC-0 4.0). 
Juno is a NASA space probe orbiting the planet Jupiter. It was built by Lockheed Martin and is operated by NASA's Jet Propulsion Laboratory. In Roman mythology, Jupiter was the king of the gods and Juno was the Queen, after whom the month June is named. The spacecraft was launched from Cape Canaveral Air Force Station on 5 August 2011 UTC, as part of the New Frontiers program. ${ }^{[6]}$ Juno entered a polar orbit of Jupiter on 5 July 2016 UTC, ${ }^{[4][}[\underline{7}]$ to begin a scientific investigation of the planet. [] After completing its mission, Juno will be intentionally deorbited into Jupiter's atmosphere. []]

Juno's mission is to measure Jupiter's composition, gravitational field, magnetic field, and polar magnetosphere. It will also search for clues about how the planet formed, including whether it has a rocky core, the amount of water present within the deep atmosphere, mass distribution, and its deep winds, which can reach speeds up to $620 \mathrm{~km} / \mathrm{h}(390 \mathrm{mph}) .{ }^{[9]}$

Juno is the second spacecraft to orbit Jupiter, after the nuclear powered Galileo orbiter, which orbited from 1995 to 2003.[]ㅡ Unlike all earlier spacecraft sent to the outer planets, ${ }^{[8]}$ Juno is powered by solar arrays, commonly used by satellites orbiting Earth and working in the inner Solar System, whereas radioisotope thermoelectric generators are commonly used for missions to the outer Solar System and beyond. For Juno, however, the three largest solar array wings ever deployed on a planetary probe play an integral role in stabilizing the spacecraft as well as generating power. $\underline{[10]}$

There are even more of it

- dfdf

- dfdfdf

- dfdf

- dfdfdf

- $\mathrm{df}^{\mathrm{df}} \mathrm{dfdf}$ dfdfdf

- [1] $]^{1}$ 


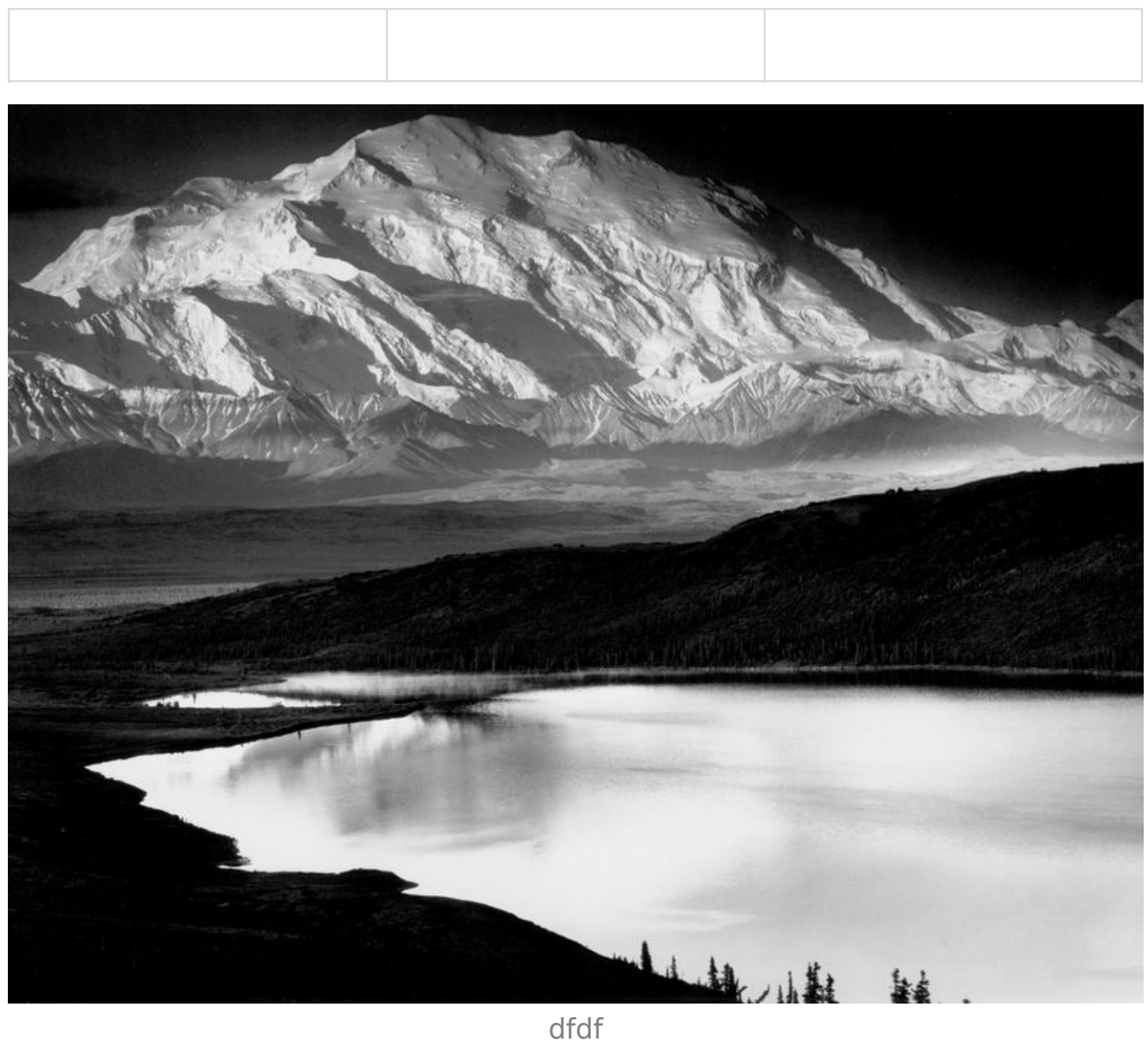

\section{Footnotes}

1. $\triangleq$

\section{Citations}

1. $\triangleq$ 